ББК 22.251

\title{
ОПЕРАТИВНАЯ ОЦЕНКА ТРЕЩИНОСТОЙКОСТИ СТАЛЕЙ
}

\author{
Юлия Сагидулловна Бахрачева \\ Кандидат технических наук, доцент кафедры физики и химии, \\ Московский государственный университет путей сообщения, Волгоградский филиал \\ bakhracheva@yandex.ru \\ ул. им. милиционера Буханцева, 48, 400120 г. Волгоград, Российская Федерация
}

Аннотация. Предложен метод прогнозирования трещиностойкости по результатам испытаний на растяжение. Предложенная модель показывает правомерность использования методов механики сплошных сред при анализе процессов, происходящих в зоне ограниченной пластичности перед фронтом трещины.

Ключевые слова: трещиностойкость, фронт трещины, кривая течения, энергия пластической деформации, испытание на растяжение.

Наличие в промышленности огромного парка оборудования с истекшим паспортным сроком службы постоянно ставит вопрос о правомерности продления срока эксплуатации. Это сложная задача, требующая наличия надежных методов оценки состояния металла в изделии. Крупногабаритные объекты, находящиеся в эксплуатации, не могут быть доставлены в лабораторию для проведения испытаний. Многие объекты работают столь долгое время, что методы расчета изделий подобного типа, а также критерии оценки пригодности к дальнейшей работе давно изменились. Это, в частности, относится к объектам, испытывающим воздействие переменных нагрузок. Последние приводят к росту трещин и вызывают опасность хрупкого или псевдохрупкого разрушения.

Наиболее современным методом оценки n склонности материалов к хрупкому разрушению

¡ При исследовании усталостных разрушений и

ос остаточного ресурса все чаще применяются

兽 методы механики разрушения $[3 ; 4 ; 6]$.

В данной статье рассмотрена возможжо. ность упрощения предложенной в работах [1;

(a) 2; 5] методики. С этой целью предложено в вычислениях использовать среднее значение величины энергии пластической деформации в расчете на единицу поверхности в малой пластической зоне протяженностью $r$ перед фронтом трещины. Для этого сначала была вычислена средняя величина интенсивности упруго-пластической деформации $\varepsilon_{i \text { ср }}$ в малой пластической зоне перед фронтом трещины:

$$
\begin{gathered}
\varepsilon_{i \mathrm{cp}}=\frac{1}{r} \int_{0}^{r} \varepsilon_{f} \exp \left[-\varepsilon_{f} x /(1,155 \cdot W)\right] d x= \\
=1,155\left(\frac{W}{r}\right) \cdot\left(1-\exp \left(-\frac{\varepsilon_{f} \cdot r}{1,155 \cdot W}\right)\right) .
\end{gathered}
$$

После этого по обобщенной кривой течения определяли величину интенсивности напряжений $\sigma_{i c p}$, соответствующую значению $\varepsilon_{i c p}$. Результаты расчетов для низкопрочных и высокопрочных сталей приведены в таблицах 1,2 .

После этого рассчитывали среднюю энергию пластической деформации на единицу поверхности в зоне ограниченной пластичности перед фронтом трещины:

$$
\gamma_{\mathrm{cp}}=\frac{\sigma_{0,2}+\sigma_{i \mathrm{cp}}}{2} \cdot \varepsilon_{i \mathrm{cp}} \cdot r .
$$


Значения величин $\sigma_{\mathrm{z}}, \varepsilon_{\mathrm{p}}$ и $\sigma_{\mathrm{cp}}, \varepsilon_{\mathrm{cp}}$ для низкопрочных сталей

\begin{tabular}{|c|c|c|c|c|c|}
\hline Сталь & $T, \mathrm{~K}$ & $\sigma_{\mathrm{B}}, \mathrm{MПа}$ & $\sigma_{\mathrm{cp}}, \mathrm{M \Pi а}$ & $\varepsilon_{\mathrm{p}}$ & $\varepsilon_{\mathrm{cp}}$ \\
\hline \multirow{4}{*}{ 10Г2ФБ } & 293 & 630 & 573 & 0,118 & 0,148 \\
\hline & 243 & 665 & 417 & 0,104 & 0,148 \\
\hline & 213 & 690 & 710 & 0,101 & 0,148 \\
\hline & 77 & 1020 & 1068 & 0,065 & 0,096 \\
\hline \multirow{4}{*}{ ВСт. 3кп } & 293 & 420 & 549 & 0,137 & 0,136 \\
\hline & 243 & 510 & 306 & 0,135 & 0,130 \\
\hline & 213 & 540 & 639 & 0,134 & 0,124 \\
\hline & 77 & 910 & 1007 & 0,068 & 0,060 \\
\hline \multirow{4}{*}{$17 Г С$} & 293 & 555 & 482 & 0,119 & 0,127 \\
\hline & 243 & 626 & 372 & 0,114 & 0,129 \\
\hline & 213 & 630 & 545 & 0,109 & 0,121 \\
\hline & 77 & 898 & 915 & 0,067 & 0,077 \\
\hline \multirow{4}{*}{$17 Г 1 C-У$} & 293 & 470 & 647 & 0,121 & 0,145 \\
\hline & 243 & 590 & 361 & 0,115 & 0,138 \\
\hline & 213 & 630 & 837 & 0,110 & 0,132 \\
\hline & 77 & 915 & 1053 & 0,067 & 0,096 \\
\hline \multirow{3}{*}{ 06Г 2НАБ } & 293 & 562 & 483 & 0,114 & 0,174 \\
\hline & 213 & 665 & 516 & 0,103 & 0,155 \\
\hline & 77 & 948 & 908 & 0,063 & 0,124 \\
\hline
\end{tabular}

Примечание. Здесь и в следующей таблице: $\sigma_{\text {в }}$ предел прочности; $\sigma_{\text {ср }}-$ средняя величина интенсивности напряжений; $\varepsilon_{\mathrm{p}}$ - величина интенсивности упруго-пластической деформации; $\varepsilon_{\mathrm{cp}}-$ средняя величина интенсивности упруго-пластической деформации.

Таблица 2

Значения величин $\sigma_{\text {в }}, \varepsilon_{\mathrm{p}}$ и $\sigma_{\mathrm{cp}}, \varepsilon_{\mathrm{cp}}$ для высокопрочных сталей

\begin{tabular}{|c|c|c|c|c|c|c|}
\hline Материал & $\begin{array}{c}t_{\text {зак }}, \\
{ }^{\circ} \mathrm{C}\end{array}$ & $\stackrel{t_{\text {отп }},}{{ }^{\circ} \mathrm{C}}$ & $\sigma_{\mathrm{B}}, \mathrm{MПа}$ & $\sigma_{\mathrm{cp}}, \mathrm{MПа}$ & $\varepsilon_{\mathrm{p}}$ & $\varepsilon_{\mathrm{cp}}$ \\
\hline \multirow{2}{*}{ 20CrMnMoVA } & \multirow[t]{2}{*}{900} & 250 & 1481 & 1396 & 0,217 & 0,148 \\
\hline & & 300 & 1457 & 1439 & 0,162 & 0,146 \\
\hline 22CrMnSiMoVA & 900 & 200 & 1543 & 1429 & 0,207 & 0,131 \\
\hline \multirow{7}{*}{ 25SiMn2MoVA } & \multirow{7}{*}{900} & 200 & 1686 & 1547 & 0,199 & 0,119 \\
\hline & & 300 & 1624 & 1535 & 0,178 & 0,121 \\
\hline & & 200 & 1821 & 1608 & 0,233 & 0,124 \\
\hline & & 250 & 1784 & 1636 & 0,214 & 0,129 \\
\hline & & 300 & 1765 & 1653 & 0,221 & 0,149 \\
\hline & & 350 & 1770 & 1659 & 0,196 & 0,129 \\
\hline & & 400 & 1717 & 1633 & 0,182 & 0,129 \\
\hline 42CrMoA & 1170 & 200 & 2019 & 1746 & 0,155 & 0,068 \\
\hline \multirow{5}{*}{ 30CrMnSiNi2A } & \multirow{5}{*}{900} & 200 & 1703 & 1521 & 0,233 & 0,110 \\
\hline & & 230 & 1676 & 1540 & 0,204 & 0,108 \\
\hline & & 250 & 1786 & 1555 & 0,304 & 0,121 \\
\hline & & 280 & 1655 & 1531 & 0,209 & 0,114 \\
\hline & & 300 & 1601 & 1514 & 0,198 & 0,121 \\
\hline \multirow{4}{*}{ CrNiMo } & \multirow{4}{*}{860} & 240 & 1754 & 1694 & 0,198 & 0,141 \\
\hline & & 290 & 1646 & 1547 & 0,245 & 0,146 \\
\hline & & 340 & 1675 & 1595 & 0,219 & 0,141 \\
\hline & & 400 & 1578 & 1529 & 0,199 & 0,144 \\
\hline
\end{tabular}

Примечание. $t_{\text {зак }}-$ температура закалки; $t_{\text {отп }}$ - температура отпуска. 
Результаты расчетов по формуле (2) для двух групп рассмотренных выше сталей показаны на рисунке 1.

Как видно на рисунке 1, зависимость $K_{I c}\left(\sqrt{ } \gamma_{\text {cp }}\right)$ также описывается для высокопрочных и низкопрочных сталей одной прямой линией:

$$
K_{I c}=232 \sqrt{\gamma_{\mathrm{cp}}}+37
$$

Результаты сравнения средних значений интенсивностей деформаций и напряжений с величинами равномерных деформаций и пределов прочности для исследуемых сталей приведены на рисунках 2 и 3.

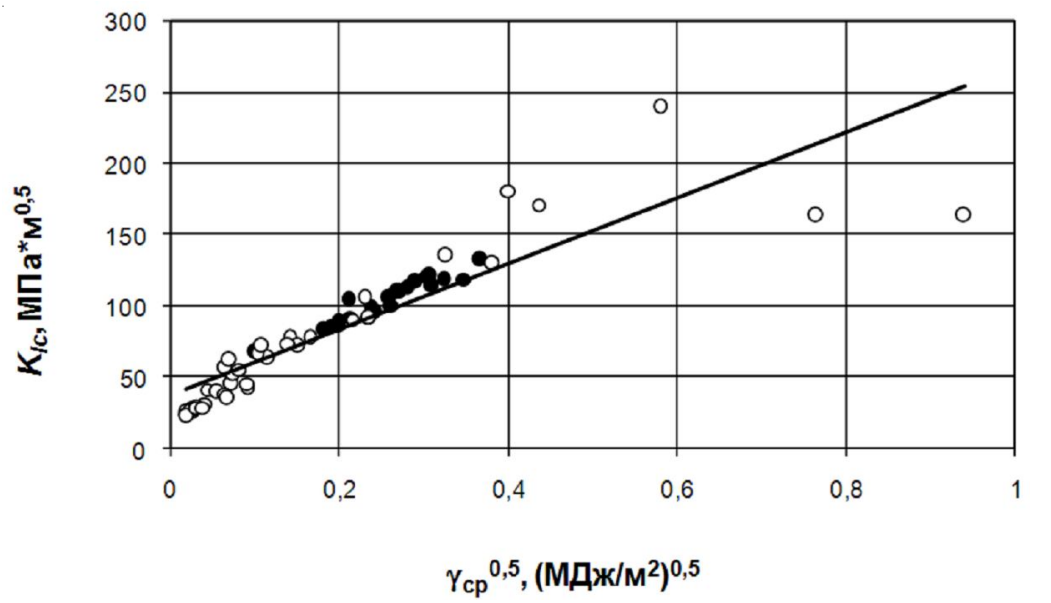

Рис. 1. Зависимость $K_{I c}$ от величины $\sqrt{ } \gamma_{\text {ср }}$ :

- - высокопрочные стали, о - низкопрочные стали

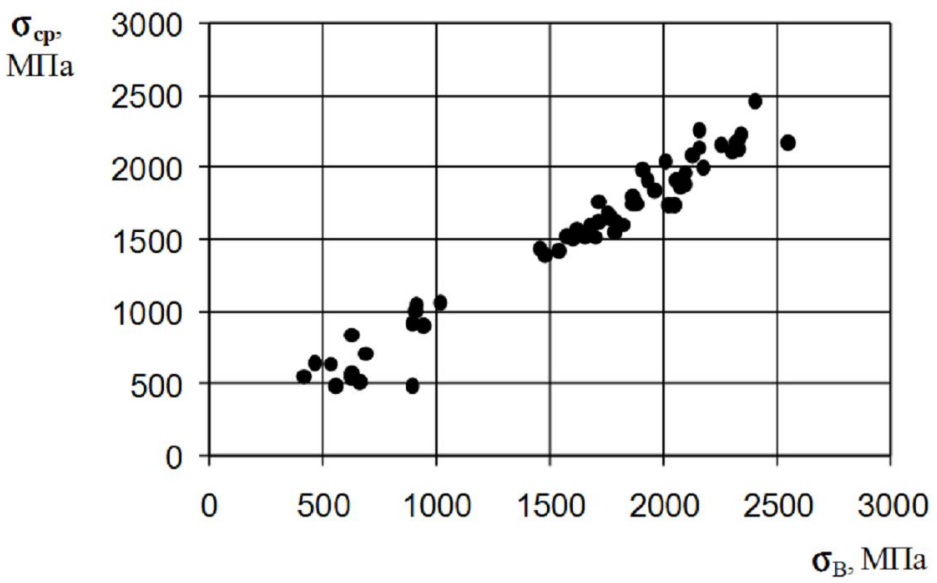

Рис. 2. Зависимость $\sigma_{\text {ср }}$ от величины $\sigma_{\text {в }}$

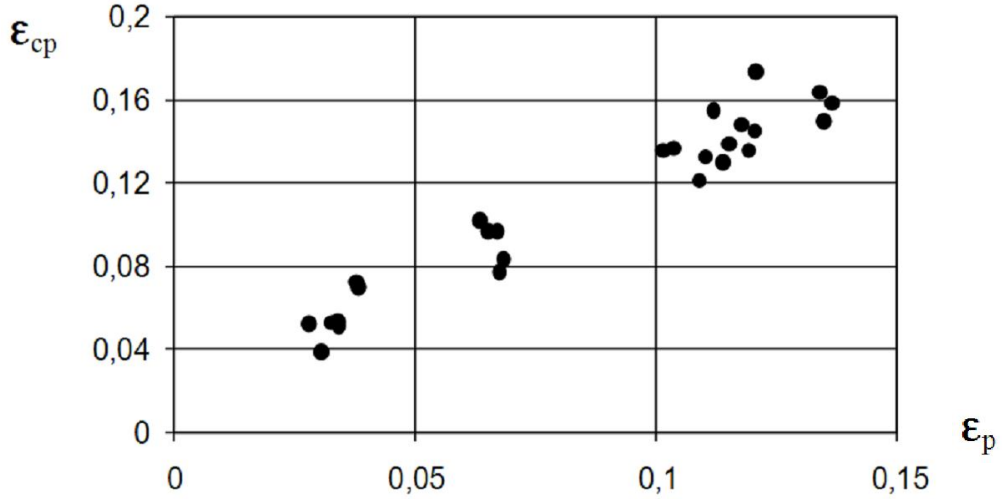

Рис. 3. Зависимость $\varepsilon_{\text {ср }}$ от величины $\varepsilon_{\text {p }}$ 


\section{ТЕХНИКО-ТЕХНОЛОГИЧЕСКИЕ ИННОВАЦИИ}

Видно, что для всех рассмотренных сталей наблюдается линейная зависимость между $\varepsilon_{i \text { ср }}$ и $\varepsilon_{\mathrm{p}}$, а также $\sigma_{i \text { ср }}$ и $\sigma_{\mathrm{B}}$. Это дает возможность привести формулу (2) к следующему виду:

$$
\gamma_{\mathrm{pas}}=\frac{\sigma_{0,2}+\sigma_{\mathrm{B}}}{2} \cdot \varepsilon_{\mathrm{p}} \cdot r
$$

Расчеты по формуле (4) дают результат, аналогичный расчетам по формуле (2) (см. рис. 4).

Как видно из рисунка 4, зависимость $K_{I c}\left(\sqrt{ } \gamma_{\text {рав }}\right)$ также линейна:

$$
K_{I c}=330 \sqrt{\gamma}+36 .
$$

Можно сделать следующие выводы:

1. Установлены закономерности соотношений трещиностойкости и удельной энергии пластической деформации слоев металла, прилегающих к поверхности разрушения, для сталей различных уровней прочности.

2. Предложен метод расчета среднего значения величины энергии пластической деформации в малой пластической зоне протяженностью $r$ перед фронтом трещины.

3. Показана линейная зависимость между $\varepsilon_{i \text { ср }}$ и $\varepsilon_{\mathrm{p}}$, а также $\sigma_{i \text { ср }}$ и $\sigma_{\text {в }}$, что позволило предложить упрощенный метод расчета энергии пластической деформации в упрочненном слое под поверхностью разрушения по стандартным механическим свойствам.

\section{СПИСОК ЛИТЕРАТУРЫ}

1. Бахрачева, Ю. С. Оперативная оценка склонности материалов к хрупкому разрушению при статическом и циклическом нагружении : дис. ... канд. техн. наук / Бахрачева Юлия Сагидулловна. Великий Новгород, 2004. - 126 с.

2. Бахрачева, Ю. С. Оценка вязкости разрушения сталей по результатам контактного деформирования / Ю. С. Бахрачева // Вестник Волгоградского государственного университета. Серия 10 , Инновационная деятельность. - 2012. - № 2. - С. 5356. - DOI: 10.15688/jvolsu10.2012.2.9.

3. Влияние содержания азота на структуру и свойства нитроцементованной стали / В. И. Шапочкин [и др.] // Металловедение и термическая обработка металлов. - 2010. - № 9. - С. 12-18.

4. Дрозд, М. С. Аналитическое исследование напряженного состояния при внедрении упругой сферы в упругопластическое полупространство / М. С. Дрозд, А. П Осипенко // Металловедение и прочность материалов : межвуз. сб. науч. тр. ВолгГТУ. - Волгоград : Изд-во ВПИ, 1975. C. $12-19$.

5. Красовский, А. Я. Трещиностойкость сталей магистральных трубопроводов / А. Я. Красовский, В. Н. Красико. - Киев : Наукова думка, 1990. -176 с.

6. Медведев, Р. С. Применение статистических методов управления качеством для оптимизации производства по ремонту пути / Р. С. Медведев, Ю. С. Бахрачева // Вестник Волгоградского государственного университета. Серия 10, Инновационная деятельность. - 2014. - № 3 (12). - С. 93-97. DOI: $10.15688 /$ jvolsu10.2014.3.11.

7. Шапочкин, В. И. Нитроцементация в условиях периодического изменения состава ат-

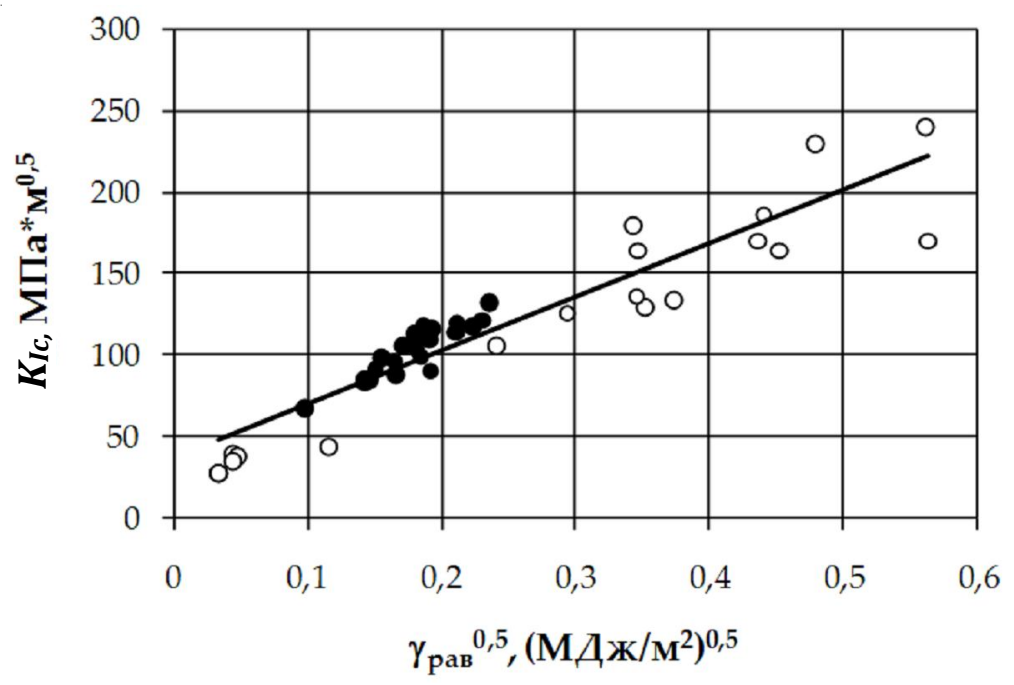

Рис. 4. Зависимость $K_{I c}$ от величины $\sqrt{ }_{\text {рав }}$ :

- - высокопрочные стали, о - низкопрочные стали 


\section{ТЕХНИКО-ТЕХНОЛОГИЧЕСКИЕ ИННОВАЦИИ}

мосферы / В. И. Шапочкин, Л. М. Семенова, Ю. С. Бахрачева // Материаловедение. - 2010. № 8. - C. 52-58.

8. Bakhracheva, Yu. S. The Method for Lifetime Estimation Through the Mechanical Properties in Tension / Yu. S. Bakhracheva // Вестник Волгоградского государственного университета. Серия 10, Инновационная деятельность. - 2014. - № 2. - С. 27 32. - DOI: 10.15688/jvolsu10.2014.2.4.

9. Valve Cam Design Using Numerical Step-ByStep Method / A.V. Vasilyev, Yu. S. Bakhracheva, O. Kabore, Yu.O. Zelenskiy // Вестник Волгоградского государственного университета. Серия 10, Инновационная деятельность. - 2014. - № 1. - С. 2632. - DOI: 10.15688/jvolsu10.2014.1.4.

\section{REFERENCES}

1. Bakhracheva Yu.S. Operativnaya otsenka sklonnosti materialov k khrupkomu razrusheniyu pri staticheskom i tsiklicheskom nagruzhenii: dis. ... kand. tekhn. nauk [Rapid Assessment of the Propensity of Materials to Brittle Fracture Under Static and Cyclic Loading. Cand. techn. sci. diss.]. Velikiy Novgorod, 2004. $126 \mathrm{p}$.

2. Bakhracheva Yu.S. Otsenka vyazkosti razrusheniya staley po rezultatam kontaktnogo deformirovaniya [Evaluation of Fracture Toughness of Steels According to the Results of the Contact Deformation]. Vestnik Volgogradskogo gosudarstvennogo universiteta. Seriya 10, Innovatsionnaya deyatelnost [Science Journal of Volgograd State University], 2012, no. 2, pp. 53-56. DOI: 10.15688/ jvolsu10.2012.2.9.

3. Shapochkin V.I., et al. Vliyanie soderzhaniya azota na strukturu i svoystva nitrotsementovannoy stali [The Influence of Nitrogen Content on the Structure and Properties of Nitrated Face-Hardened Steel]. Metallovedenie i termicheskaya obrabotka metallov, 2010, no. 9, pp. 12-18.

4. Drozd M.S., Osipenko A.P. Analiticheskoe issledovanie napryazhennogo sostoyaniya pri vnedrenii uprugoy sfery $\mathrm{v}$ uprugoplasticheskoe poluprostranstvo [Analytical Investigation of the Stress State at Introduction of Elastic Sphere in ElasticPlastic Half-Space]. Metallovedenie $i$ prochnost materialov: mezhvuzovskiy sbornik nauchnykh trudov VolgGTU [Metal Science and the Durability of Materials: Interuniversity Collection of Scientific Works of Volgograd State Polytechnic University]. Volgograd, Izd-vo VPI, 1975, pp. 12-19.

5. Krasovskiy A.Ya., Krasiko V.N. Treshchinostoykost staley magistralnykh truboprovodov [Crack Resistance of Steels of Main Pipelines]. Kiev, Naukova dumka Publ., 1990. 176 p.

6. Medvedev R.S., Bakhracheva Yu.S. Primenenie statisticheskikh metodov upravleniya kachestvom dlya optimizatsii proizvodstva po remontu puti [The Use of Statistical Methods of Quality Management to Optimize Production on Track Repair]. Vestnik Volgogradskogo gosudarstvennogo universiteta. Seriya 10, Innovatsionnaya deyatelnost [Science Journal of Volgograd State University. Technology and Innovations], 2014, no. 3 (12), pp. 9397. DOI: $10.15688 /$ jvolsu10.2014.3.11.

7. Shapochkin V.I., Semenova L.M., Bakhracheva Yu.S. Nitrotsementatsiya v usloviyakh periodicheskogo izmeneniya sostava atmosfery [Nitrocarburizing in the Conditions of Periodic Changes in Atmospheric Composition]. Materialovedenie, 2010, no. 8, pp. 52-58.

8. Bakhracheva Yu.S. The Method for Lifetime Estimation Through the Mechanical Properties in Tension. Vestnik Volgogradskogo gosudarstvennogo universiteta. Seriya 10, Innovatsionnaya deyatelnost [Science Journal of Volgograd State University. Technology and Innovations], 2014, no. 2, pp. 27-32. DOI: 10.15688/jvolsu10.2014.2.4.

9. Vasilyev A.V., Bakhracheva Yu.S., Kabore O., Zelenskiy Yu.O. Valve Cam Design Using Numerical Step-By-Step Method. Vestnik Volgogradskogo gosudarstvennogo universiteta. Seriya 10 , Innovatsionnaya deyatelnost [Science Journal of Volgograd State University. Technology and Innovations], 2014, no. 1, pp. 26-32. DOI: 10.15688/ jvolsu10.2014.1.4.

\section{RAPID ASSESSMENT OF FRACTURE TOUGHNESS OF STEEL}

\section{Yuliya Sagidullovna Bakhracheva}

Candidate of Technical Sciences, Associate Professor,

Department of Physics and Chemistry,

Moscow State University of Railway Engineering (Volgograd Branch)

bakhracheva@yandex.ru

Bukhantseva St., 48, 400120 Volgograd, Russian Federation 


\section{ТЕХНИКО-ТЕХНОЛОГИЧЕСКИЕ ИННОВАЦИИ}

Abstract. The presence in industry of a huge Park of equipment with expired passport life constantly poses the question of the justification of the lifetime extension. This is a complex task that requires reliable methods for assessing the state of metal in the product. Large objects, which are in operation, cannot be delivered to the laboratory for testing. Many objects are so long that the methods of calculation of products of this type, as well as criteria for evaluating the suitability for further work changed long ago. This, in particular, is experiencing the effects of variable loads. The latter lead to growth of cracks and pose a risk of brittle fracture or pseudographs. The modern method for assessing the propensity of a material to brittle fracture is to determine the fracture toughness.

The method is proposed for predicting the fracture toughness according to the results of the tensile tests. The proposed model shows the validity of using methods of continuum mechanics in analysis of the processes taking place in the area bounded plasticity in front of the crack.

The regularities of the relationships of fracture toughness and specific energy of plastic deformation of layers of metal adjacent to the fracture surface, for steels of different strength levels.

Key words: fracture toughness, crack front, curve of flow, energy of plastic deformation, tensile test. 